\title{
Name Code
}

National Cancer Institute

\section{Source}

National Cancer Institute. Name Code. NCI Thesaurus. Code C93600.

A coded value specifying the non-unique textual identifier for the entity. 